\title{
Germanica
}

\section{Du roman sentimental à la littérature féminine érotique contemporaine}

\section{Le renouvellement d'un genre}

From Sentimental novels to contemporary erotic literature for women. The renewal of women's writing

Vom Liebesroman zur zeitgenössischen trivialen Frauenliteratur. Die

Erneuerung eines Genres

\section{Régine Atzenhoffer}

\section{(2penEdition}

\section{Journals}

Édition électronique

URL : http://journals.openedition.org/germanica/2741

DOI : 10.4000/germanica.2741

ISSN : 2107-0784

\section{Éditeur}

Université de Lille

\section{Édition imprimée}

Date de publication : 30 décembre 2014

Pagination : 211-227

ISBN : 9782913857346

ISSN : 0984-2632

Référence électronique

Régine Atzenhoffer, " Du roman sentimental à la littérature féminine érotique contemporaine », Germanica [En ligne], 55 | 2014, mis en ligne le 30 décembre 2016, consulté le 06 octobre 2020. URL http://journals.openedition.org/germanica/2741 ; DOI : https://doi.org/10.4000/germanica.2741 


\title{
Du roman sentimental à la littérature féminine érotique contemporaine
}

\author{
Le renouvellement d'un genre
}

\author{
Régine ATZENHOFFER \\ Université de Strasbourg
}

En Allemagne, à l'aube du XXI ${ }^{e}$ siècle, la littérature féminine populaire, jusque-là traditionnellement sentimentale, est devenue érotique. On pourrait voir dans cette nouvelle écriture féminine et son dire érotique, un double défi : s'emparer d'un sujet longtemps tabou et occuper un territoire précédemment réservé aux hommes. Éros est dans l'air, à notre ère. Vit-il un âge d'or? Si l'on considère le déploiement de l'érotisme dans la littérature féminine populaire contemporaine et les articles et émissions consacrés à ce phénomène ${ }^{1}$, on peut y voir, sans nul doute, une période faste. Distribuée longtemps sous le manteau, la fiction érotique s'est démocratisée et évolue à l'avenant, en expansion et en évidence sur les étalages et dans les vitrines. Quant au marché du livre, la production et la consommation de ce type d'écrits tiennent le haut du pavé et les libraires contribuent à sa considérable diffusion. Nous assistons donc

1. - «Die Zukunft des Buches : Erotische Literatur » in : FAZ, 12.02.2013; «Erotische Literatur sorgt für einen heißen Herbst » in : Stern, 3.11 .2003 ; « Neue FrauenLiteratur: Sie schreiben, wollen Sex und sind wütend » in : Die Welt, 22.01.2009; «Die neuen Erotikromane von und für Frauen modernisieren das Genre » in : Hannoversche Allgemeine, 2.02.2010 ; «Erotik-Roman ist der Verkaufsschlager » in : SchwarzwälderBote, 21.12.2012 ; «Erotische Frauenliteratur verkauft sich gut» in : Hannoversche Allgemeine, 18.07.2012 ; « Erotische Literatur boomt » in : WDR, Die frauTV-Lesung, 8.05.2014. 
à une profonde transformation de la littérature féminine populaire en Allemagne, où les scripts permettent l'apparition d'une subjectivité désirante au féminin. Une des conséquences de cette parole féminine est d'abord linguistique : plus de dentelles pour nimber le désir comme à l'âge classique du roman d'amour obsédé par les bienséances et le happy end. L'héroïne, objet désiré dans la littérature féminine traditionnelle, se mue très souvent en sujet désirant : nous sommes loin ici du « conte de fées pour adultes » d'auteures telles que Wilhelmine Heimburg, Eugenie John Marlitt et Hedwig Courths-Mahler qui, comme Wothe ou Lehne (pseudo pour Helene Butenschön [note de la rédaction]), ont suscité bien des vocations ${ }^{2}$ et fait véritablement charnière dans l'histoire du roman féminin populaire allemand, actuellement en plein renouveau. Un renouveau dût notamment à des choix marketing et éditoriaux plus osés et à des romans qui, à l'instar de Feuchtgebiete ${ }^{3}$ de Charlotte Roche, transgressent des tabous auparavant circonscrits à l'espace privé. Scatologique et pornographique, ce premier roman de Charlotte Roche, publié en 2008, déclenche une immense médiatisation en Allemagne 4 . Plaidoyer féministe ou banale arnaque commerciale? Manifeste de combat féministe ou « roman gynéco-porno de gare » d'une animatrice de télé non conventionnelle 5 ? Faut-il soupçonner derrière ce succès, l'aboutissement d'une stratégie marketing 6 ? C'est bien ce que

2. - Gertrud Willenborg : « Das ist die Welt der rechten Adelsmenschen », in : Kölnische Rundschau, 9.07.1961 : «Längst hat die Autorin Nachfolger und Nachfolgerinnen gefunden, die zwar weniger berühmt sind, aber ebenso gern gelesen werden. Diese Nachfolge ist nicht auf Romanheftserien beschränkt, sondern auch Illustriertenromane und viele Filme gehen den gleichen Weg ».

3. - Charlotte Roche : Feuchtgebiete, Köln, DuMont, 2008.

4. - À titre d'exemple : Stuttgarter Zeitung, 21.08.2013 : «Bei Charlotte Roche, so meint sich fast jeder zu erinnern, der nicht zu den 2,5 Millionen Buchkäufern gehört, wird das Publikum zum Tampon, zum Analzäpfchen, zur Klobürste im Leben eines pubertierenden Mädchens, es wird durch jeden Dreck, Schleim und Sabber gezogen, den ein Körper nur hergeben kann, es wird in Urin mariniert, mit Blut bebackpinselt und mit Erbrochenem paniert. Wohl bekomm's! »; Westdeutsche Zeitung, 10.03.2008 : «Feuchtgebiete handelt von Pimmel, Muschi und Aua »; Berliner Zeitung, 26.08.2013: « Die Sozialisation sei schuld, bürgerliche Körper- und Moralvorstellungen. Und diese würden gekränkt, wenn eine Frau - wie eben in den Feuchtgebieten - plötzlich über ihre Körperflüssigkeiten spricht, gerne mit Sperma spielt und ihren Tampon mit ihrer besten Freundin, der Blutsschwester, tauscht. Es geht hier um Körperflüssigkeiten, die ganz dezidiert als exkremental beschrieben werden. Ein Gegenentwurf zur Hochglanzversion von Weiblichkeit ».

5. - Charlotte Roche s'est d'abord fait connaître grâce à l'émission Fast forward, diffusée par la chaîne musicale VIVA2. Présentatrice télé sur des chaînes touchant un public de niches comme le magazine Tracks d'Arte dans sa version allemande, elle a reçu le Prix Adolf Grimme et le Prix Bavarois du cinéma. Elle est une figure déjà connue du monde médiatique lors de la parution de son premier roman au titre racoleur.

6. — « Mit kalkuliertem Skandal zum Megaseller?» in: Stern, 7.08.2011. 
laisse entendre le quotidien Frankfurter Rundschau. Le fait est que ce livre séduit les uns et irrite les autres. Et le statut de phénomène social qui lui est conféré explique la foire médiatique qu'il déclenche et les nombreuses interrogations qu'il soulève : est-ce un ouvrage pornographique ? Doit-on le lire comme un pamphlet radical dénonçant le culte forcé de la beauté et de l'hygiène au féminin dans une société où le rappel omniprésent à l'image de soi est devenu tyrannique ? Est-ce là une contribution supplémentaire à un nouveau féminisme très controversé, décrit dans Neue Deutsche Mädchen ${ }^{7}$ ou Wir Alphamädchen ${ }^{8}$ ? D'aucuns croient reconnaître en Charlotte Roche le porte-drapeau d'une nouvelle génération d'auteures en Allemagne, où, un demi-siècle après l'entrée en vigueur de l'égalité juridique entre hommes et femmes, on assiste à une poussée de l'écriture érotique féminine. À la suite de la parution de Feuchtgebiete et de Schoßgebete ${ }^{9}$, son second opus dont les vingt premières pages décrivent une fellation et la dernière une sodomie, cette nouvelle tendance à l'exhibitionnisme radical, à la description de pratiques sexuelles extrêmes, à l'eldorado orgasmique prôné par des auteures contemporaines à succès dont Sandra Henke, Astrid Martini, Kerstin Dirks ou Lilly Grünberg, sonne-t-il le glas du roman sentimental et de sa légendaire pudeur?

\section{Une auteure féministe délurée ragaillardit la littérature féminine populaire allemande}

Vingt-sept pays ont acquis les droits de Feuchtgebiete, publié en Allemagne en février 2008. Depuis, cet ouvrage de Charlotte Roche est un véritable phénomène de société outre-Rhin où il a été vendu à plus d'un million et demi d'exemplaires. À la veille de sa parution en France, Arte a consacré un documentaire à l'histoire de ce livre polémique. Hélène Memel, 18 ans, hospitalisée à la suite d'une fissure anale, se remémore ses prouesses sexuelles et revient par le détail sur ses habitudes de vie: elle s'amuse à s'enfoncer un noyau d'avocat dans le sexe, s'abstient de se laver afin de décupler le fumet de ses odeurs corporelles ou se parfume le creux des oreilles avec ses sécrétions vaginales. Rien n'est épargné au lecteur, de ses hémorroïdes à ses fluides corporels, de sa technique de la «culotte coupée », de sa rencontre avec un homme qui passe une heure tous les samedis à la raser des pieds à la tête, de sa cicatrice anale tout juste opérée qu'elle rouvre pour rester hospitalisée

7. - Jana Henkel / Elisabeth Raether: Neue Deutsche Mädchen, Berlin, Rowohlt, 2008.

8. - Meredith Haaf / Susanne Klingner / Barbara Streidl: Wir Alphamädchen, Hamburg, Hoffmann \& Campe, 2008.

9. - Charlotte Roche: Schoßgebete, München, Piper, 2013. 
plus longtemps et ainsi laisser plus de temps à ses parents divorcés de se réconcilier. Feuchtgebiete est scandaleux, dégénéré, et il est assumé comme tel par son auteure. Elle nomme les humeurs, dit les odeurs, déclame les saveurs :

Wenn ich mit jemandem ficke, trage ich doch mit Stolz sein Sperma in allen Körperritzen, an den Schenkeln, am Bauch oder wo der mich sonst noch vollgespritzt hat [...] Ich mag es gerne, wenn Sperma auf der Haut trocknet, Krusten bildet und abplatzt. Wenn ich mit meiner Hand einen Schwanz wichse, achte ich immer darauf, dass etwas Sperma an meinen Händen bleibt. Das kratze ich mit meinen langen Fingernägeln auf und lasse es darunter hart werden, um es später am Tag als Andenken an meinen guten Fickpartner mit den Zähnen unter den Nägeln rauszuknabbern, im Mund damit rumzuspielen, drauf rumzukauen und es nach langem Schmecken und Schmelzenlassen runterzuschlucken. Das ist eine Erfindung, auf die ich sehr stolz bin: Mein Sexandenkenkaubonbon ${ }^{10}$.

Sa parole s'exprime à travers tous ses orifices et cite tour à tour le sperme, le pus, le sang, les excrétions, les sécrétions. Charlotte Roche ne fait pas seulement l'éloge du corps et de ses fonctions. Elle parle d'un corps qui sent et coule. Elle s'oppose à sa déréalisation, à sa sanctification et, surtout, à l'hygiénisme en mettant en question l'image véhiculée par les magazines et la publicité. Les femmes sont obsédées par la propreté et par l'idée de se débarrasser de toutes leurs excrétions, explique-t-elle. D'où l'idée d'écrire sur les parties du corps humain qui dégoûtent ${ }^{11}$. Assez de ces corps de femmes aseptisés, il faut du poil et des propos cochons, tel est son credo. Au départ, précise-t-elle dans le documentaire diffusé sur Arte, elle pensait rédiger un texte revendicatif, un pamphlet sur les femmes allemandes, sur leur rapport avec leur « minette ». F. Beigbeder, qui dans le même documentaire d'Arte évoque le culte de la nature cher aux Allemands, fait référence aux mouvements nudistes et hygiénistes du début du siècle. Mais, puisqu'elle dénonce justement ce culte du corps et ses performances, l'approche de Charlotte Roche en est diamétralement opposée.

Ce qui ressort de Feuchtgebiete, c'est la dénonciation tacite de l'hygiène, dénonciation récupérée par les féministes en Allemagne pour mettre en avant l'avilissement par la société de la femme dans son rapport à son corps et à ses fluides. Ce roman a fait fureur en Allemagne de par son côté politiquement incorrect, son langage cru et son propos

10. - Charlotte Roche: Feuchtgebiete, op. cit., p. 26.

11. - Ibid., p. 9-10: « Da bei mir der Arsch offensichtlich zum Sex dazugehört, ist er auch diesem modernen Rasurzwang unterworfen [...] Ich weiß im Gegensatz zu anderen Menschen sehr genau, wie mein Poloch aussieht. Ich gucke es täglich in unserem Badezimmer an ». 
subversif. Le témoignage profane d'une jeune femme adepte d'une sexualité débridée, à $70 \%$ autobiographique selon l'auteure, contient tous les éléments propres à créer un malaise chez le lectorat. Le penchant pour la provocation de Charlotte Roche s'est soldé, au-delà des expérimentations anales et vaginales de l'hérö̈ne Hélène, par une proposition sans ambiguïté : le président fédéral, Christian Wulff, s'est vu proposer une nuit avec l'auteure, membre du collectif Attac et opposante à la mondialisation, s'il refusait de ratifier la loi concernant les centrales nucléaires déjà validée par les députés allemands. Charlotte Roche est devenue du jour au lendemain l'icône d'un « féminisme pop », la figure de proue d'un féminisme d'avant-garde qui assume une sexualité décomplexée ${ }^{12}$. Feuchtgebiete, la négation des plus élémentaires leçons d'hygiène, est un pamphlet anti-hygiéniste, un pied de nez à la culture de la beauté parfaite prônée par Heidi Klum et ses consœurs de l'émission Germany's next top-model. Scandale, écœurement, engouement: son premier ouvrage a propulsé Charlotte Roche au-devant de la scène médiatique. En Allemagne, ce genre de récit qui parle d'excréments, de sexe, de saleté et de liberté, qu'il soit féministe ou non, est novateur. Force est de constater que dans d'autres pays européens, la cochonnerie littéraire post-moderne est bien moins représentée qu'en France. Mais en therme de provocation, Charlotte Roche surpasse largement Catherine Millet. À la première lecture du manuscrit de Feuchtgebiete, M. Hartges, des éditions DuMont, pensait même tenir là un livre underground: c'est devenu un roman populaire et son auteure un phénomène d'édition outre-Rhin.

Cet ouvrage à la couverture rose fuchsia est-il une compilation de scènes pornographiques? Charlotte Roche n'est aucunement outragée qu'on l'accuse de pornographie. Dans une interview au Spiegel notamment, elle déclare que son récit n'est pas uniquement réservé aux femmes, mais aussi destiné aux hommes. La pornographie utilise des codes calibrés pour arriver à une proposition qui soit très rapidement la plus stimulante possible. Or son livre ne cherche pas à entraîner le lecteur sur la pente de sa libido et à l'exciter. Il y a, chez l'auteure, une volonté de polémiquer et non de provoquer. Une polémique vive qui pose les termes d'un débat, celui du corps de la femme-objet. Elle fustige, dans plusieurs interviews, les féministes de la vieille garde dont Alice Schwarzer qui voit dans la pornographie une haine à l'encontre des femmes. Les femmes aussi aiment la pornographie, affirme Charlotte

12. - Die Welt, 22.01.2009: «Neue Frauen-Literatur [...] Mit billigen Sexromanen haben sie nichts zu tun. Vielmehr etablieren sie ein neues, freizügiges Frauenbild in der Literatur. Es gibt Neues von der Rolle der Frau in der Gesellschaft. Zumindest in der Literatur. Dort blüht eine sexuelle Befreiung mit seltsamen Vorzeichen [...] Denn wir erleben eine zweite Welle des ambitionierten Frauenromans nach den Siebzigern ». 
Roche. Il leur manque juste une langue pour exprimer leur désir. Ne doit-on pas admettre ce langage vulgaire de la bouche d'une femme? Feuchtgebiete tient du manifeste, et les aînées de l'auteure s'appellent Valérie Solanas et Virginie Despentes. Charlotte Roche s'inscrit dans la filiation de Scum manifesto et Baise-moi, et son roman marque son époque tout autant que l'histoire des femmes et celle de la littérature féminine populaire en Allemagne ${ }^{13} .2008$ témoigne donc d'un tournant dans l'histoire de cette littérature: Feuchtgebiete, en tête des ventes, supplante la chick-lit et les romans sentimentaux de Christiane Sadlo, édités sous le pseudonyme Inga Lindström, qui ont, depuis 2004, généré des recettes colossales en librairie et sur le petit écran, les producteurs y voyant l'opportunité d'attirer à la fois les lecteurs et les non lecteurs. Un certain lectorat - selon l'enquête du magazine Ster ${ }^{14}$ des femmes entre 20 et 30 ans, des hommes de plus de 50 ans et des jeunes couples -, lassé par la fadeur des romans d'amour, se tourne vers des lectures plus polémiques, plus dérangeantes. Cette évolution du goût, surtout d'un lectorat jeune et féminin, engendre ce qui peut être considéré comme un renouveau de la littérature féminine populaire. Le cru, le brut s'imposent, et à côté des récits de séduction apparaît la littérature érotique, voire pornographique, et de domination.

\section{Des moiteurs d'un pamphlet anti-hygiéniste à une littérature érotique investie par les femmes}

Feuchtgebiete, mais plus encore Schoßgebete, sont des œuvres de transition et Charlotte Roche le maillon d'une chaine allant du roman rose au roman érotique, en passant par la chick-lit, la mom-lit et la bit-lit. Même si l'engouement du lectorat féminin pour ce type de récits peut être assimilé à un phénomène de mode, Charlotte Roche et, en 2012, E. L. James ont contribué à l'avènement d'un nouveau sous-genre, le roman féminin érotique, et contribué à décomplexer une parole sexuelle féminine via des récits où elles donnent libre cours à un imaginaire érotique singulier. Les romans osés de Sandra Henke ${ }^{15}$ et Astrid Martini16, publiés en 2007 et 2008, n'ont véritablement connu de succès commercial qu'après la sortie de Feuchtgebiete. La libération des mœurs et des corps a affranchi la parole sensuelle, et la littérature érotique, hier maudite, est, à compter de 2008, branchée. Le sexe a colonisé le roman

13. - Ibid.: «Das auf Ekel getrimmte Buch der Moderatorin Charlotte Roche war allerdings für Deutschland ein Startschuss [...]».

14. - Publiée le 22 avril 2008.

15. - Sandra Henke: Loge der Lust, Köln, Mira-Verlag, 2007; Opfer der Lust, Köln, Mira-Verlag, 2008 ; Lotusblüte, Hamburg, Plaisir d'Amour, 2008.

16. - Astrid Martini: Zuckermond, Hamburg, Plaisir d'Amour, 2006; Mondku $\beta$, Hamburg, Plaisir d'Amour, 2007. 
féminin populaire de langue allemande, et le désir de connaître toutes les facettes de la sexualité impose aux auteures d'être plus exploratoires, plus audacieuses, ce d'autant plus que l'écriture érotique et pornographique se banalise du fait de la poussée du X et de l'explosion du trash sur le Net. Depuis 2010, la littérature érotique féminine engrange ainsi de sérieux succès en Allemagne comme en témoigne la réussite commerciale de Jazz Winter, Ina Paul, Lilly Grünberg, Nina Jansen ou Lucy Palmer (qui publie aussi sous le pseudonyme Inka Loreen Minden). Ce qui frappe dans la multitude de ces textes graveleux, c'est la suprématie des écrits féminins, ce qui prouve qu'il existait une attente longtemps déçue d'une vraie littérature érotique féminine. Depuis la libération sexuelle, l'écriture féminine mute pour devenir plus verte, pour s'affranchir notamment de la bienséance, de la pudeur et du bon goût qui l'ont longtemps garrottée. Si l'histoire de la littérature érotique a toujours été riche d'auteurs féminins, ces dernières étaient souvent tapies sous le masque de pseudonymes trompeurs. Les femmes libres du passé ont pris la plume pour évoquer, dans des œuvres légères, la vie secrète du sexe faible, les rêveries de la jeunesse enfermée au couvent, le frisson de la pucelle au seuil de la nuit de noces. Mais, dans une société où il a longtemps été inconvenant d'évoquer tout ce qui relève de l'intimité, il est rare que ces ouvrages aient été téméraires. À présent, ils semblent bien fades, tout comme leur jaquette, comparés à ceux que l'acheteur potentiel trouve en rayon et sur les sites marchands. Il est normal qu'après des siècles de silence, les femmes, encouragées par le mouvement post-féministe en Allemagne, se soient senties appelées à s'exprimer, à dire et écrire leurs fantasmes. Force est de constater qu'une large part des auteurs du deuxième sexe s'est essentiellement illustrée dans ce genre plus ou moins neuf qu'est le « joui-dire ${ }^{17}$ ».

Par la libération de la parole et celle des pratiques sexuelles, les auteures sont devenues plus égrillardes : leurs écrits traitent à présent du sexe en termes crus, évoquent des expériences souvent peu communes dans une langue facile d'accès et primesautière. Le corps nu y est banalisé par son omniprésence: les pudeurs du discours ont disparu. Cet exhibitionnisme s'explique tout à la fois par le désir de satisfaire le voyeurisme du lectorat et le plaisir d'offrir son texte comme on donne son corps. " Nous voici arrivés à l'âge masturbatoire de la littérature », écrit Xavier Deleu ${ }^{18}$. Le désir est compulsif, le sexe un besoin pressant. Les héroïnes délurées de la littérature post-moderne se libèrent de leurs pulsions charnelles ${ }^{19}$. Dans les scènes de copulation, tout se passe

17. - Christine Détrez / Anne Simon: À leur corps défendant. Les femmes à l'épreuve du nouvel ordre moral, Paris, Seuil, 2006, p. 42.

18. - Xavier Deleu: Le Consensus pornographique, Paris, Mango, 2002, p. 47.

19. - Lilly Grünberg: Verführung der Unschuld, München, Heyne, 2010, p. 161 : 
comme si, pour affirmer la verdeur du désir féminin, les auteures se sentaient obligées de détailler la mécanique de l'acte sexuel:

Dann öffnete er rasch seine Hose, und Sina rutschte zur Seite, damit er seinen Schwanz befreien konnte. Er hatte ein wirklich schönes Stück. Groß. Kräftig. Die Eichel schimmerte rot, und ein Lusttropfen hatte sich bereits auf ihr gebildet [...] Langsam umschlossen ihre Lippen seine Eichel, saugten sich an ihr fest, und seine Spitze wurde noch heißer, glühte förmlich. Ganz vorsichtig glitten ihre Lippen tiefer, eroberten Millimeter für Millimeter des prächtigen Schafts, der in ihrem Mund zu zucken begann [...] Inzwischen hatte sie seinen Schwanz vollständig aufgenommen. Sie spürte seinen Hodensack an ihren Lippen, das Kitzeln seiner Haare. Langsam fuhr ihr Mund wieder hoch, um dann erneut an ihm hinabzugleiten, und mit jedem Mal wurde sie dabei schneller [...] Bruno kam, und es war gewaltig in ihrem Mund ${ }^{20}$.

\section{Le BDSM21, passage obligé vers le triomphe des sentiments?}

Quand il s'agit de chercher de nouveaux lecteurs devant le poste de télévision et le PC, l'édition, les romancières et la littérature féminine populaire s'adaptent à la nouvelle explosion des fétichismes et des obsessions. Dans la société contemporaine où la femme assume ouvertement ses pulsions, se livre aux pires turpitudes sans craindre les foudres d'un ordre moral désormais révolu, une nouvelle époque s'ouvre et donne une force noire au sexe. Le récit lubrique féminin, écrasé par la banalisation du sexe, est obligé d'aller aux extrêmes pour espérer frapper les esprits, appâter le chaland et parvenir à lutter contre la concurrence des images des films $\mathrm{X}$ et des sites web trash. Ne restait donc plus qu'à trouver le moyen, par des œuvres aguicheuses, de toucher un public de plus en plus large. Ainsi, la « littérature-viande » se déleste de ce qui est trop complexe pour aller à l'essentiel et plonge dans des passions fortes pour en finir avec les plaisirs fades. Les succès de l'écriture érotique féminine en Allemagne aujourd'hui sont la preuve du triomphe simultané du «cul et du cucul », de la chick-lit et du trash. Les jouissances de l'héroïne sont inséparables d'une sexualité violente et du sadisme de l'amant qui multiplie les expériences lubriques pour l'emmener au cœur de pratiques à sensations fortes ${ }^{22}$. Le roman féminin érotique est entré dans l'ère de

\footnotetext{
« So etwas absolut Verruchtes Tabuloses, Lasterhaftes wollte sie auch erleben! "Mach endlich weiter, warum machst du nicht weiter?", quietschte sie atemlos [...] "Bitte", wimmerte sie, "bitte, nimm mich, es kommt mir gleich, [...] bitte, bitte !" ».

20. - Kerstin Dirks: Schülerin der Lust, Berlin, Ullstein, 2013, p. 64-65.

21. - Bondage, Punition, Sadisme, Masochisme.

22. - Sandra Henke: Im Schatten der Lust, München, Heyne, 2012, p. 33 : «Bondage. Rollenspiel. Erziehung. Dominanz und Unterwerfung. Spanking. Flagellation.
} 
la spécialisation: latex, BDSM, bondage, etc. Aucun insolite sexuel n’y est omis.

Il y a là une nouvelle écriture érotique féminine, celle de la douleur et de la cruauté, d'une sexualité de la domination et de la soumission qui s'inscrivent en faux contre les évolutions libérales des sociétés démocratiques. Une mise en écriture d'expériences sexuelles débridées et une pratique à haute dose d'une « obscénité fleur bleue » mêlent mots doux et termes orduriers. Avant, de telles scènes, passées sous silence, existaient en creux dans les récits; à présent, elles occupent des chapitres entiers. Comparés au récit grivois classique, ces textes féminins qui dévoilent les jeux érotiques extrêmes auxquels une femme peut s'adonner pour plaire à son amant et entretenir sa flamme, sont encore neufs :

Ihre Waden waren an die Oberschenkel gebunden und ihre Arme hinter dem Kopf verschränkt und fixiert, so dass sie vollkommen schutzlos war. Besonders ihre Intimstellen. Jemand hatte sie verschnürt wie ein Paket, wobei er jedoch ihre Brüste und ihre Mitte ausgespart hatte. Die Ansätze ihres Busens waren mit einem dünnen Seil umwickelt, so dass die Brüste wie kleine Türme nach oben ragten und die Spitzen wie Gipfelkreuze anmuteten ${ }^{23}$.

La littérature érotique féminine allemande sombre dans l'Éros noir: c'est la nouvelle mode des amours vaches. Le goût pour le sadisme, la perversion et les fessées saturent les chapitres de ces romans dont les titres, Rausch der Unterwerfung (Cornelia Eden), Bestrafe mich ! (Nina Jansen), Unterworfen (Lena Morell), Arie der Unterwerfung (Ivy Paul), Viola: das Tagebuch der Sklavin (Sira Rabe), Die Geschichte einer Leibeigenschaft (Sisa) ou encore Absolute Hingabe (Jazz Winter), disent tout du contenu. Plus les romancières se disent libérées, plus leurs héroïnes s'empressent de clamer leur attachement à la virilité dominatrice 24 . Il suffit de regarder les titres et les jaquettes de ces livres pour prendre la mesure des nouveaux penchants. On devine que ce ne sont pas

Bastinade. SM ». Voir aussi Lilly Grünberg: Verführung der Unschuld, op. cit., p. 216: «Es war unglaublich aufregend, zwei Männern gleichzeitig ausgeliefert zu sein, ihre Hände überall zu spüren, sich ihnen nicht entziehen zu können, selbst wenn sie es gewollt hätte ».

23. - Sandra Henke: ibid., p. 32-33. Astrid Martini : Feuermohn, Berlin, Ullstein, 2009, p. 199: « Die Wellen des Schmerzes waren noch nicht abgeklungen, da folgte ein weiterer Tropfenregen [...] Sie hielt den Atem an, blickte wie hypnotisiert in die flackernde Flamme, die am umliegenden Wachsrand züngelte und weitere Tropfen entkommen ließ. Unbarmherzig fielen sie auf Anna nieder. Diesmal auf ihre Brüste ».

24. - Astrid Martini : Feuermohn, op. cit., p. 82: «Das war es, was sie heimlich ersehnte: Härte, Dominanz, Gehorsam und Unterwerfung ». Lilly Grünberg: Verführung der Unschuld, op. cit., p. 29: «Ihr Verstand wehrte sich gegen die Unterdrückung, aber ihr Körper war anderer Meinung und gab sich ganz der Lust hin ». 
là des apologies de la tendresse ou des odes aux vertus du Blümchensex, la « sexualité vanille ». Les spice girls de l'écriture parlent du rapport homme-femme comme d'un rapport sujet-objet et affirment la soumission ou le masochisme des hérö̈nes qui, loin d'être en révolte contre l'ordre masculin, sont des poupées dociles. Elles ont intégré pour ellesmêmes les pulsions phallocrates du sexe fort : «Sie sehnte sich nach purer Dominanz ${ }^{25}$. [...] Sie wollte ihm bedingungslos gehorchen $»^{26}$. Les passions nouvellement banalisées pour des pratiques hier encore honteuses aboutissent au morcellement des corps dans l'imaginaire érotique. On assiste au découpage symbolique de l'anatomie féminine au service du plaisir masculin ; le corps féminin n'est que la somme de segments de chairs sélectionnés à l'aune d'une sexualité phallique et éjaculatoire.

Une série d'épreuves obligent l'héroïne à repousser loin les limites de ce qu'elle pense être capable d'endurer. Les scènes s'enchaînent ; elle est tour à tour attachée, fessée et fouettée. Trouvant l'extase dans la douleur, elle jouit par le fait même de montrer à son amant et maître qu'elle peut tout supporter pour lui, qu'elle peut tout endurer au nom de leur lien. Elle jouit de la douleur et tire vanité du désir qu'elle inspire à son maître. Elle accepte tous les outrages, tous les sévices, elle les espère même comme autant de défis. Elle repousse toujours plus loin ses propres limites en connaissant des plaisirs forts, interdits au commun des mortels:

Ohne eine Antwort abzuwarten, richtete er sich auf und begann sie mit der Gerte zu züchtigen. Anna schrie und zuckte. Schmerz fuhr durch ihren Körper. Diese Schläge hatten nichts mit den Hieben gemeinsam, die sie kurz zuvor hatte genießen dürfen. Sie waren grausam und unerbittlich [...] Aaron kannte kein Erbarmen, ließ die Gerte wieder und wieder auf sie niedersausen, ließ kaum einen Zentimeter ihres Körpers aus. Das harte Leder prasselte immer und immer wieder auf ihren Bauch, ihre Brüste, ihre Arme und Beine, dann dazwischen und schießlich auf die zarteste Stelle ihres Körpers. Ihre gellenden Schreie ignorierte er, betrachtete stattdessen zufrieden die roten Striemen auf ihrer blaßen Haut. Und dann nahm die Wucht der Schläge ab. Sie wurden gezielter gesetzt, gefühlvoll platziert. Anna begann sich zu beruhigen, und obwohl ihre Haut brannte, begann sie die einzelnen Hiebe bald nicht mehr als Schmerz, sondern als lüsternes Kribbeln zu empfinden. So intensiv, dass sie es nicht erwarten konnte, mit weiteren Schlägen bedacht zu werden. Sie ersehnte die Momente, in denen sich

25. - Astrid Martini : ibid., p. 72.

26. - Ibid., p. 86. Voir également ibid., p. 164: « Das alles ertrug sie freiwillig [...] Nahm diesen Kelch auf sich, weil er es so wollte ». Lilly Grünberg: Verführung der Unschuld, op. cit., p. 195: "Ihre Haut brannte und entlockte ihr jammernde Laute, zugleich fühlte sie eine unerklärliche Zufriedenheit darüber, ihm ausgeliefert und gehorsam zu sein ». 
der Schmerz in ein süßes Gefühl verwandelte, von Mal zu Mal intensiver, bis sie trunken vor Ekstase und benebelt vor Hingabe war ${ }^{27}$.

En réalisant le pouvoir de son corps, elle tire sa force de la fascination qu'elle exerce sur son amant qui, lui, cherche perpétuellement à se montrer digne de sa proie en lui faisant miroiter de nouveaux raffinements dans le supplice. Le maître et son esclave sont, dans ces récits, soudés l'un à l'autre par leurs pulsions. La jeune femme s'oppose, dans un premier temps, à son amant pour finalement accéder à toutes ses demandes. Tombée sous la coupe d'un homme qui délaisse les pratiques conventionnelles et la somme de se livrer à des activités SM, elle y trouve une affirmation de soi. En lui interdisant, pendant un certain laps de temps, le plaisir, l'amant cherche à vivre le fantasme du viol; il veut jouir de son pouvoir, l'avoir à ses pieds et soumise. Le sexe se révèle plus que jamais le lieu de tous les défoulements. Si la mise en scène SM est typique de la littérature trash contemporaine, si les viols, les aventures plurielles etc..., ont toujours existé dans la littérature lubrique, ces activités hors normes ont changé de statut en même temps que les écrits, émaillés de travers misogynes en contradiction complète avec le féminisme ardent, ont évolué et que les envolées anciennes des féministes sont oubliées. Alors qu'ils apparaissent comme inédits de la part d'une romancière, ces textes, s'ils étaient signés par un homme, seraient attentatoires à la dignité féminine. À la volonté de puissance de l'amant, les romancières contemporaines n'opposent rien de neuf : elles n'offrent aucune ébauche d'une sensualité nouvelle et inventive.

Comment expliquer que leurs héroïnes soient prêtes à faire les quatre volontés de celui qu'elles appellent leur maître ? Elles pensent à le quitter plusieurs fois mais ne peuvent s'y résoudre. N'arrivant pas à faire abstraction des liens sensibles, des transports affectifs et des inclinations personnelles, elles rêvent toutes d'une génitalité non découplée des oscillations du cœur ${ }^{28}$. Ce qui les pousse donc à se soumettre à la

27. - Astrid Martini : ibid., p. 103-104. Sandra Henke: Im Schatten der Lust, op. cit., p. 128-129: « Der Schmerz hallte in ihr wider, aber da Sam mit der Gerte von hinten über ihre Spalte rieb, verschmolzen Lust und Pein auf köstliche Weise. Dieses neue Gefühl war unbeschreiblich, sie sehnte sich danach, es noch einmal zu erleben [...] Erstaunlicherweise blieb die Lust, obwohl er sie nicht mehr liebkoste, sondern nur noch traktierte. Allein der Schmerz erregte Naomi auf bittersüße Art und Weise [...] Es schien ihr, als würden die Schläge ihren Körper aufwecken. Sie hatte sich noch nie so wach, so verankert im Hier und Jetzt und so lebendig gefühlt ».

28. - Lilly Grünberg: Verführung der Unschuld, op. cit., p. 234 : «Zu gerne hätte sie gefragt: Lieben Sie - liebst du mich?; denn dies war das Einzige, was ihr für das vollkommene Glück fehlte - eine nicht nur körperliche, sondern auch von wahren Gefühlen dominierte Verbundenheit ». Ibid., p. 267: «Aber obwohl Guilias Körper weiterhin nach diesen erotischen Liebesspielen lechzte und auf wunderbare Weise befriedigt wurde, genügte ihr dies nach den Tagen der intensiven Nähe und Vertraulichkeit nicht mehr 
dictature du fouet notamment, c'est l'amour qu'elles éprouvent pour leur maître. Il s'agit de lui montrer que nulle douleur ne leur fait peur quand il s'agit de prouver leur affection. Nous sommes en fait en présence d'un roman sentimental, dans lequel l'abnégation de l'hérö̈ne éclate à chaque page. Elle se donne dans l'espoir de déclencher des ardeurs et faire naître des sentiments chez ce maître qui, lui, demande sans fin de nouvelles preuves d'attachement qui requièrent chaque fois l'avilissement de la jeune femme. En se soumettant aux pires caprices SM de son amant, la maîtresse timorée, la plus farouche des jeunes filles, se mue invariablement en une Messaline enfiévrée ${ }^{29}$. Et cet empressement du deuxième sexe à se soumettre aux pulsions masculines devient burlesque à force d'être répétée. Les héroïnes cherchent à connaître les beautés du sexe brut et les grâces du sentiment, à passer par les allées du coït pour espérer découvrir le paradis des amours auprès d'hommes qui semblent, souvent, avoir besoin de se venger du désir prédateur qu'elles éveillent en eux. Dans ces œuvres, le sexe est célébré sous toutes ses formes, l'orgasme est magnifié, les ivresses du plaisir sont chantées de page en page ${ }^{30}$. Le désir y est certes souverain, mais la quête amoureuse, toujours tapie au creux du texte, existe en arrière-plan. Cette écriture féminine du sexe est déconnectée de la question génésique. L'héroïne est en quête du plaisir effréné et non de la beauté de la maternité. Il semble que la coupure entre sexualité et procréation est consommée et que les romancières défendent le droit d'occuper le terrain de la violence et de l'agressivité comme les hommes. L'amour étant préempté par le roman rose, les auteures évoquent le sexe pour espérer, par le verbe, faire sentir qu'aimer fait battre les cœurs et frémir les ventres. Sexe et sentiment ne sont pas radicalement différents: les aventures débridées décrites avec moult détails (goût du sperme, éloge du membre viril, etc.) et les pratiques échangistes, le butinage galant ${ }^{31}$, ont pour fonction de renforcer l'attachement de l'hérö̈ne à son maître.

[...] Ihr Herz wollte mehr ».

29. - Ibid., p. 220 : « Sie verstand selbst nicht, woher ihre Gier nach so viel sexueller Befriedigung kam, aber ihr Körper war heiß und unersättlich ». Sandra Henke : Im Schatten der Lust, op. cit., p. 79: «Bald ritt sie Chad ausgelassen wie eine wilde Furie ».

30. - Sandra Henke: Im Schatten der Lust, op. cit., p. 111: "Welch ein Mörderorgasmus! ». Kerstin Dirks: Schülerin der Lust, op. cit., p. 60: «Was für ein geiler Orgasmus!». Astrid Martini : Feuermohn,op. cit., p. 201: «Es erfüllte sie mit grenzenloser Ekstase ».

31. - Lilly Grünberg: Verführung der Unschuld, op. cit., p. 262 : « Niemals wäre sie auf die Idee verfallen, dass es sich derart wunderbar anfühlen würde, zwei Männer auf einmal zu verwöhnen und gleichzeitig von ihnen verwöhnt zu werden ». Astrid Martini: Feuermohn, op. cit., p. 28: «Als sie jedoch den Mann erkannte, der nicht weit von ihr entfernt im Begriff war, zwei Damen gleichzeitig zu beglücken, zuckte sie zusammen », p. 68: «Ich stehe darauf, meinem Freund beim Sex mit fremden Frauen zuzusehen »; Sandra Henke: Im Schatten der Lust, op. cit., p. 60 : «Eine Ménage à trois ist nur ein 
Et, le maître n'est pas celui que l'on croit. Il n'existe et ne trouve sa place ou sa signification que par rapport à l'esclave. Il est en réalité l'esclave de son esclave, de l'acceptation de sa soumise à subir les sévices qui l'excitent lui aussi ${ }^{32}$. Les renversements de situation montrent que l'héroïne, loin de se soumettre, est en train, par l'esprit, de s'affranchir de la tutelle du maître au moment même où elle prétend l'aimer sans réserve. Quand bien même le sexe est survalorisé, il reste subordonné au sentiment chez ces romancières du « X-elles », finalement très fleur bleue. Les récits sont à la fois des apologies du sexe pur et des hymnes à l'amour. Les romancières et les héroïnes sont ainsi prises dans une sorte de grand écart entre dévergondage et grand amour, entre pudeur et volupté, le tout en contradiction avec la littérature leste traditionnelle qui, elle, a toujours insisté sur la mécanique des corps bien plus que sur les ressorts du sentiment. L'érotisme classique masculin n'a jamais cherché à agrafer le sentiment au sexe: il a toujours été entendu que cette littérature a pour fonction d'émoustiller et non d'émouvoir. La nouvelle Ève de l'écriture épicée, au moment même où elle tisse des récits tous plus verts les uns que les autres, ne peut renoncer à dépeindre l'espérance de l'amour et du happy-end. Car derrière les récits les plus débridés de la sexualité féminine contemporaine se cache, plus puissant que jamais, le mythe du grand amour de la littérature rose :

Er liebte sie, er brauchte sie - er wollte sie. Mit ihr und keiner anderen wollte er sein Leben verbringen und Kinder großziehen [...]. „Ja, ich liebe dich, und ich wollte es dir schon lange sagen“", antwortete er leise [...] Es war wie im Märchen ${ }^{33}$.

À l'instar des romans roses, le roman féminin populaire à caractère érotique débute par la rencontre des protagonistes qui scelle la naissance du couple. La part la plus conséquente du volume textuel est ensuite consacrée aux diverses disjonctions, qu'elles soient d'ordre interne (doute, angoisse, etc.) ou externe (suite d'aventures sexuelles, malentendus, etc.). Et, autre constante, le dénouement est heureux. Le roman d'amour « établit [...] que l'infortune amoureuse ne peut être que transitoire, qu'elle mène un passage obligatoire vers le triomphe des sentiments partagés $»^{34}$. Le roman féminin égrillard reprend cette même trame bien que les protagonistes soient d'un type différent. Si le premier se conclut nécessairement par un mariage et, pour les plus éche-

echter Dreier, wenn sie mit einem Sandwich endet ».

32. - Astrid Martini : ibid., p. 245-246: «Er begehrte sie und hatte für diesen Tag ursprünglich etwas ganz anderes mit ihr im Sinn gehabt als dieses Peitschenspielchen [...] Er wollte sie riechen, spüren, ausfüllen [...] Wollte von ihr kosten. Jetzt ».

33. - Ibid.

34. - Alain-Michel Boyer: La Paralittérature, Paris, PUF, 1992, p. 105. 
velés, par un chaste baiser, le second - un récit extrêmement codé dont toutes les problématiques d'ordre politique ou social ont été évincées et facilement identifiable grâce à sa couverture - n'hésite pas à mettre les corps en scène de manière très explicite.

Le roman érotique féminin est bel et bien un avatar novateur du roman sentimental traditionnel: il a su adapter son contenu afin d'être en adéquation avec le monde contemporain. La clé du succès du récit féminin de l'étreinte réside en un juste dosage entre le SM et le côté fleur bleue. Et cette version hot de Cendrillon évoque le mélange de deux imaginaires, celui du X et celui du sitcom. D'aucuns attribueront ceci à la difficulté d'innover dans un domaine par définition immuable. Non seulement la mécanique des corps n'a pas varié depuis les origines, mais les imaginaires érotiques qui ont nourri les littératures légères du libertinage comme celles du $\mathrm{XX}^{\mathrm{e}}$ siècle sont globalement restés les mêmes. Pour réussir à imposer une écriture originale, il faut un imaginaire hors norme, une fantasmagorie singulière qui est le propre des grands auteurs. Y a-t-il néanmoins des procédés spécifiques de l'écriture de l'érotisme au féminin? Sarane Alexandrian et Francesco Alberoni ${ }^{35}$ voient, tous deux, de notables différences entre les textes lubriques féminins et masculins: l'homme étant davantage animé par des rêves de possession alors que celui du deuxième sexe est supposé faire davantage la part belle au désir de fusion, les textes «masculins » seraient plus dans la recherche de l'efficacité, dans l'envie d'aller à l'essentiel. La progression du désir que l'on trouve dans l'écriture féminine n'intéresserait pas les hommes ; seules les romancières décrivent tout le chemin qui mène au lit. Quand le masochisme devient, comme ici, sentimental, il correspond bien plus au code du roman sentimental qu'à celui du véritable roman érotico-pornographique.

\section{La Fick- und Fotziliteratur, des fictions fleurant la recette}

On aurait pu imaginer que la libération des mœurs et l'émancipation des femmes allaient signer l'essoufflement du roman sentimental, mais la bluette, récit à finalité purement récréative, se porte toujours bien. S'intéresser au devenir du roman d'amour sans développer le poids économique et éditorial du « roman sentimental à caractère érotique », ce serait sans doute passer outre un de ses éléments constitutifs: son aspect commercial. En 2013, les ventes en Allemagne de ces romans à « consommation nonchalante $»^{36}$ mettant en scène tous les stéréotypes dont les féministes essaient, depuis plusieurs années, de se débarrasser

35. - Sarane Alexandrian: Histoire de la littérature érotique, Paris, Seghers, 1989 ; Francesco Alberoni : L'Érotisme, Paris, Ramsay, 1987.

36. - Concept établi par Richard Hoggart. 
mais qui plaisent aux lectrices, atteignaient près de 9 millions de titres. De plus, les adaptations au cinéma ${ }^{37}$ contribuent à la popularité du genre renouvelé et à lui conférer une visibilité certaine. La palette de l'érotisme littéraire féminin de langue allemande est aujourd'hui extrêmement riche et le roman féminin érotique a gagné plusieurs points de part de marché, ce qui incite les commerçants du livre, acteurs de cette mutation littéraire, des plus connus comme Rowohlt, Ullstein, Heyne, Blanvalet ou Goldmann (sauvé, en 2012, du dépôt de bilan par la publication de la version allemande du best-seller de E. L. James, Fifty Shades of Grey), aux plus audacieux, dont Schwarzkopf \& Schwarzkopf (avec sa série Anais) ou MIRA Taschenbuch, à diffuser les nouveautés mutines. D'autres éditeurs, dont Plaisir d'Amour, Elysion et Blue Panther Books, ont tout misé sur l'écriture féminine érotique et tout fait pour devenir les références dans ce domaine : ils sont devenus les leaders dans le domaine de la littérature de charme en Allemagne. Ils ont plongé l'écriture graveleuse sans hésiter et chassent sur les terres de la publication gaillarde par opportunisme. Devant le succès de Charlotte Roche, éditée en 2008 par DuMont, d'autres maisons comme Piper et Random House du groupe éditorial Bertelsmann, contraintes de se diversifier, se sont ouvertes aux textes corsés pour faire face au marché émergeant du livre électronique et du support numérique. Bertelsmann, von Holtzbrink et Springer, les géants de l'édition en Allemagne, ont ainsi lancé, parallèlement à leurs collections classiques, des avatars sulfureux du roman sentimental. Pour gagner des parts de marché, ils visent un cœur de cible, la lectrice entre 20 et 50 ans, et la segmentation, rend facilement identifiables les différentes collections. Cette poussée de la publication érotique féminine s'explique donc, en premier lieu, par la stratégie éditoriale des maisons d'édition qui décident de publier ce qui fait recette chez les concurrents. Ainsi, des auteures comme Sandra Henke et Sophie Andresky sont publiées chez plusieurs éditeurs.

La flambée de l'écriture grasse est donc tout sauf spontanée. Si elle correspond à une véritable libération de la parole, elle est surtout le fruit d'un empressement éditorial pour tout ce qui touche au sexe en raison des succès rencontrés par les pionnières de l'écriture grivoise contemporaine. Du fait de la mutation du lectorat, les responsables de publication cherchent des artifices pour faire venir à la littérature ceux que la télévision et internet ont attirés. Pour ce faire, la littérature en rajoute dans l'obscène et le graveleux pour espérer séduire ceux que les livres d'ordinaire laissent froids. Ainsi est apparue, en Allemagne, une nouvelle littérature répétitive, calibrée pour plaire au plus grand nombre. Les éditions de poche ou de format semi-poche avec des couvertures expli-

37. - Feuchtgebiete, par exemple, est sorti sur grand écran en août 2013. 
cites inondent de récits érotiques les librairies et autres points de vente du livre. On ne peut occulter les millions de lectrices, dont 20 à $25 \%$ seraient en fait des lecteurs, et leur engouement pour ces livres et leur version électronique, les ebooks. Les maisons d'édition font preuve de pragmatisme avec leur site internet. Le tournant numérique a été abordé en proposant une large offre de titres sur une plateforme d'achat en ligne: acheter en ligne un roman sentimental, érotique, représente des avantages majeurs dont la garantie, relative, de l'anonymat et une offre quasi instantanée. Si l'on tient compte du circuit de distribution en ligne et des circuits parallèles comme le prêt entre particuliers, bien que ces modes d'acquisition soient difficilement quantifiables, on perçoit bien l'ampleur de ce type de littérature qui circule d'autant plus que sa valeur vénale est faible.

Les romans féminins populaires contemporains, hypersexualisés, relèvent bien d'une production à grande échelle qui, de par certaines de ses caractéristiques, peut être assimilée à la notion d'industrie culturelle telle qu'elle a été définie par les philosophes de l'École de Francfort. En d'autres termes, leur écriture intègre parfaitement, dès leur création, la finalité d'atteindre des consommateurs dans un but essentiellement lucratif. Comment expliquer alors que les écrits sulfureux de Mia Ming, Ariadne von Schirach, Astrid Martini ou Sandra Henke ${ }^{38}$, publiés entre 2006 et 2008, n'aient pas connu le succès fulgurant et planétaire de Cinquante nuances de Grey? La littérature pornographique à l'eau de rose allemande, publiée avant l'engouement pour le roman d'E. L. James, n'a pas fait l'objet d'un succès fabriqué: ni traduction en langues étrangères, ni affiches publicitaires, ni produits dérivés ${ }^{39}$, ni émissions, ni séances de dédicace, ni articles de presses, ni curiosité attisée par le bouche à oreille, ce qui est, dans une démarche de communication commerciale, une première marche vers l'envol des ventes d'un livre-buzz planétaire. Il aura fallu Feuchtgebiete et Cinquante nuances de Grey pour ouvrir une brèche pour le roman érotique féminin outreRhin. La lecture de ces romans aux amours tortueuses, remodelés à la saveur du sadomasochisme, attise les réflexions autour de la littérature post-féministe allemande et des nouvelles générations d'auteures dont les hérö̈nes se plaisent à mettre à bas les tabous et à exalter le sexe. De femmes-objets, ces dernières sont devenues aujourd'hui des femmessujets qui construisent et assument, via leur sexualité, leur manière

38. - Mia Ming: Schlechter Sex, Berlin, Schwarzkopf \& Schwarzkopf, 2008 ; Ariadne von Schirach: Tanz um die Lust, Müchen, Goldmann, 2007; Astrid Martini : Zuckermond, op. cit.; Sandra Henke: Lotusblïte, op . cit.

39. - Sex-toys, bijoux, décoration et livres qui tentent de décrypter le roman (Le décodeur de 50 nuances de Grey), qui surfent sur la vague de l'érotisme et du SM (50 nuances de plaisir ou 50 secrets de volupté) ou des parodies (50 nuisances de Glauque). 
d'être au monde. Affranchies des luttes qui ont mobilisé leurs mères et leurs grands-mères, et du stigmate de l'infériorité longtemps attribué à leur sexe, ces auteures ont le sentiment d'être libres de choisir leur existence, leur carrière, leur manière de s'approprier leur corps et celui de vivre leur sexualité. On peut néanmoins se demander si le libre arbitre est devenu pour les femmes un fardeau dont elles voudraient se délivrer par des fantasmes de soumission ou s'il faut y voir, au contraire, un signe de la liberté d'accéder enfin, sans complexes, à leur propre pornographie. Or, qu'il soit le signe de l'échec ou du triomphe du féminisme, ce qui frappe dans cet érotisme post-féministe, c'est à quel point il est dominé par le modèle de la littérature sentimentale. 
